\title{
Unsupervised Segmentation of Synthetic Aperture Radar Sea Ice Imagery Using A Novel Markov Random Field Model
}

\author{
David A. Clausi and Huawu Deng \\ Department of Systems Design Engineering \\ University of Waterloo \\ Waterloo, Ontario, Canada N2L 3G1 \\ Email: \{dclausi,h2deng\}@engmail.uwaterloo.ca \\ Telephone: (519) 888 - 4567 x2604 Fax: (519) 746-4791
}

\begin{abstract}
The operational segmentation of SAR sea ice imagery is a practical, challenging objective in the realm of applied pattern recognition. This research is in support of operational activities at the Canadian Ice Services (CIS), a government agency that monitors all ice-infested regions under Canadian jurisdiction. This paper uses a fusion of tone and texture to segment SAR sea ice images in an unsupervised manner. A novel Markov random field (MRF) segmentation technique is employed and produces improved results over K-means and the traditional MRF implementation.
\end{abstract}

\section{INTRODUCTION}

The research literature contains many examples of the application of pattern recognition techniques to remote sensing imagery. However, few of these examples directly satisfy a defined operational need. This paper discusses an application of pattern recognition applied to remote sensing imagery with a demonstrated operational basis. This research is in support of the activities of the Canadian Ice Service (CIS) (http://www.cis.ec.gc.ca/), a government agency that is responsible for the monitoring of all iceinfested waters of Canada.

Sea ice monitoring is critical for Canada, given its northern latitude coupled with extensive ocean-based coastal regions. These regions must be monitored to satisfy tactical needs with respect to ship navigation and ice breaker priorities and to satisfy environmental assessment needs with respect to evaluating ice volumes, extents and types. The only means of producing timely information is through the use of remotely sensed imagery. Also, given the uncertain weather conditions in coastal zone regions coupled with the desire to capture imagery day or night, it is preferable to use synthetic aperture radar (SAR) for such an application. RADARSAT is a Canadian owned and operated satellite that carries a SAR sensor. The sensor was designed with ice reconnaissance as its primary target market. It uses a $5 \mathrm{~cm}$ wavelength and $H H$ polarization. Many different modes exist that encompass a variety of incidence angles and pixel resolution. The SCANSAR mode is routinely used at CIS at a resolution of $50 \mathrm{~m}$. Operationally, this data is $2 \times 2$ block averaged which does not damage its visible interpretation.

An example of a SAR sea ice image and its corresponding ice map produced at CIS is presented in Fig. 1. Given that this SAR image is dimensioned to $11,152 \times 17,632$ (pixels), the details of the ice typing are not apparent. At full resolution, details are provided that allow the ice analyst to create the ice map. The ice map indicates regions of consistent ice types. An "egg code" describes each of the regions identified in the SAR image. These egg codes are representative of a World Meteorological Organization standard (see http://www.natice.noaa.gov/egg.htm for details) used in ice typing worldwide. The first row of the egg code represents the total ice concentration (in tenths), the second row represents the ice concentration for each of the constitutive ice types, the third row indicates codes for the particular ice type, and the fourth row represents codes for the floe size.

\section{BACKGROUND}

The objective of typical ice typing algorithms in the research literature is to identify solidified ice stages, namely first year rough, first year smooth, and multiyear ice types. However, this is quite limited with respect to the true scope of the operational requirements. For example, open water takes on different forms depending on incidence angle and prevalent winds, making it very difficult to classify. Ice types in the early stages of development can mimic characteristics in later stages of development. SAR sea ice imagery is complex due to both sensor (eg. speckle noise, antenna gain patterns) and environmental (eg. ice type transitions, prevailing weather conditions) characteristics. The ice analyst uses a variety of cues for determining the ice types of a particular region, primarily tone, texture and floe boundary shape.

The primary shortcoming of the ice maps as a source of information is that each egg code region only gives regional approximations of the ice concentrations. In other words, the ice map product does not provide information 

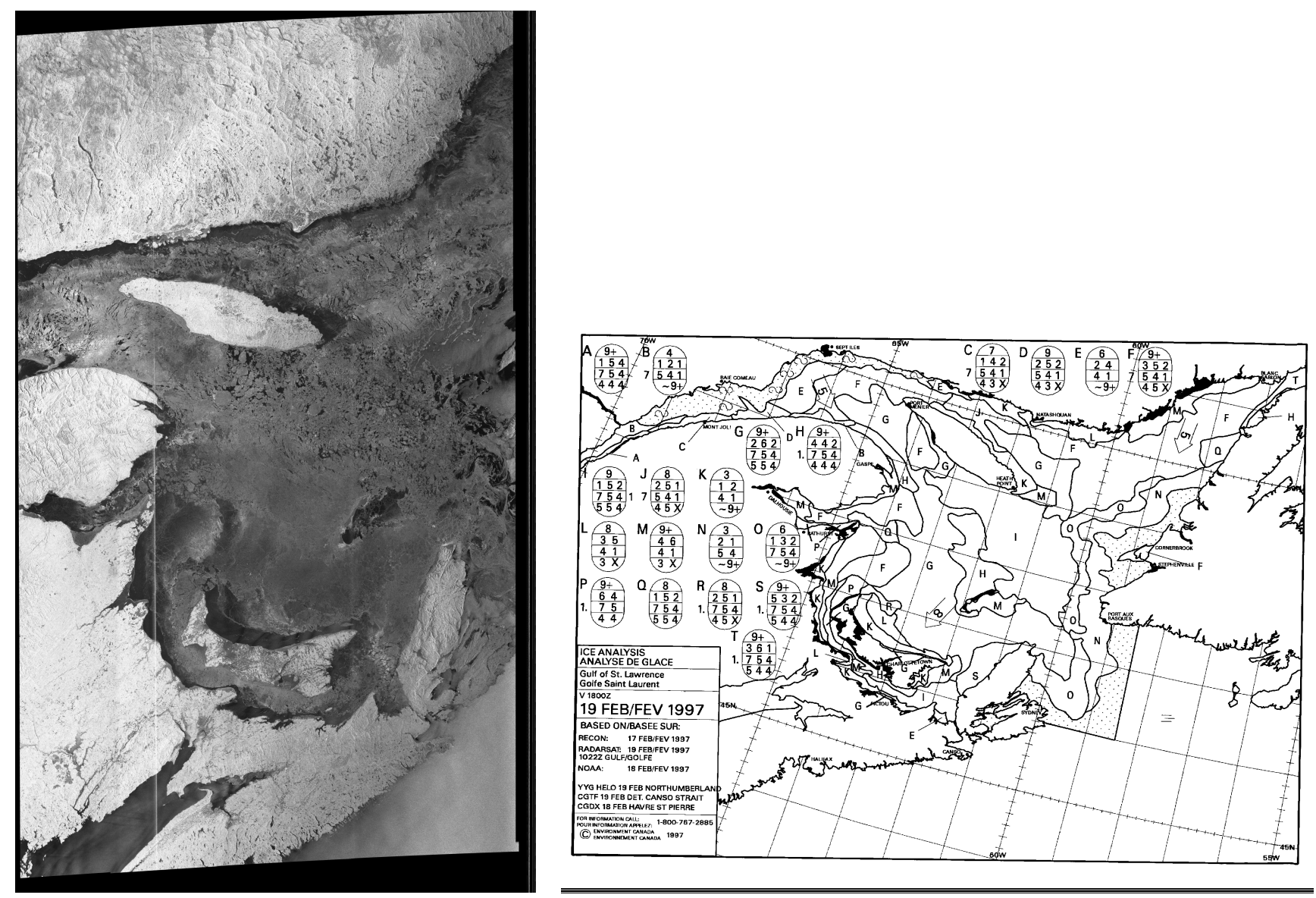

Fig. 1. RADARSAT SCANSAR sea ice image of the Gulf of St. Lawrence taken February 19, 1997 (dimensions 11,152 × 17,632 pixels). Corresponding ice map produced by Canadian Ice Services (CIS).

on a pixel-by-pixel basis. Given the vast geographical size of egg code regions, the concentrations do not provide sufficient navigational information. For example, one may know that a particular egg code region has approximately $20 \%$ multiyear ice, but there is no information to tell the ship's navigator where the multiyear is concentrated in a region that typically extends for hundreds of kilometers.

The objective in this research is to use the egg code data as input for performing pixel-by-pixel segmentation of thee associated SAR region. That is, for example, one would know a priori the number of classes as well as the labels for these classes. Although the egg codes provide an appropriate starting point for the segmentation, it is wellrecognized that these offer only approximate estimates of the a priori probabilities.

\section{SAR SEA ICE Segmentation}

\section{A. Texture Features}

Texture is a very important cue in the human visual system. Texture features have a demonstrated ability to support image segmentation in many areas and have also demonstrated potential for classifying sea ice types in SAR imagery [1] [2] [3] [4]. Various texture methods are found in the research literature to extract texture features [5]. For SAR sea ice image classification, there is supportive evidence that the gray level co-occurrence probability (GLCP) method [6] is an effective method to generate appropriate texture features [1] [2] [3] [7] [4].

Details of this algorithm are widely available and described in the above references. With respect to the testing performed here, we captured the probabilities [1] by quantizing to 64 grey levels, used a window size of $7 \times 7$, a pixel separation of 1 , and standard orientations of 0,45 , 90, and 135 degrees. Two statistics were applied to the 
probabilities to capture the texture features: contrast and entropy. This generates an 8-dimensional feature space.

Generally, the co-occurrence feature response can be modelled by a Gaussian distribution function. Denote the feature vector extracted from a random image $(X=x)$ by $F=f$, where $F$ denotes a random variable and $f$ is an instance of $F . Y=y$ stands for a segmented result based on the feature vector $F=f$. That is,

$$
p\left(f_{s}^{k} \mid y_{s}=m\right)=\frac{1}{\sqrt{2 \pi \sigma_{m}^{k}}} \exp \left[-\frac{\left(f_{s}^{k}-\mu_{m}^{k}\right)^{2}}{2 \sigma_{m}^{k^{2}}}\right],
$$

where $\mu_{m}^{k}$ and $\sigma_{m}^{k}$ are the mean and standard deviation for the $m$-th class in the $k$-th feature component, and $f_{s}^{k}$ is the $k$-th feature component of $f$ at site $s$.

\section{B. Classification}

Standard pattern recognition methods for performing clustering or classification do not account for neighbour relationships that are critical for solving a segmentation problem. For example, the standard K-means [8] approach clusters based only on the feature space representation, completely ignoring the spatial interactions. That is, a model that accounts for the high probability that adjacent pixels belong to the same class should be used.

A Markov random field (MRF) is recognized to be a powerful stochastic tool used to model the joint probability distribution of the image pixels in terms of local spatial interactions [9] [10] [11]. Using MRF models for image segmentation has a couple of advantages. First, the spatial relationship can be seamlessly integrated into a segmentation procedure. Second, the MRF based segmentation model can be inferred in the Bayesian framework which is able to utilize different types of image features.

\section{Segmentation Model}

According to the Bayes rule, the segmentation problem is formulated as:

$$
P(Y=y \mid F=f)=\frac{p(F=f \mid Y=y) P(Y=y)}{p(F=f)} .
$$

$P(Y=y \mid F=f)$ is the posteriori probability of $Y=y$ conditioned on $F=f \cdot p(F=f \mid Y=y)$ denotes the probability distribution of $F=f$ conditioned on $Y=y$ and functions to fit the feature data, which is thus referred to as the feature modelling component. $P(Y=y)$ is a priori probability of $Y=y$ and is used to describe the label distribution of a segmented result only, which is normally referred to as the region labelling component. $p(F=f)$ is the probability distribution of $F=f$. It will be assumed that each component of $F=f$ be independent on the other components with respect to $Y=y$ (conditional independence).

Suppose the energy form of $P(Y=y)$ is $E_{R}$ and that of $\prod_{k=1}^{K}\left[p\left(f^{k} \mid Y=y\right)\right]$ is $E_{F}$. A general energy form $E$ for $P(Y=y \mid F=f)$ can be derived from the product of $P(Y=y)$ and $\prod_{k=1}^{K}\left[p\left(f^{k} \mid Y=y\right)\right][10]$ :

$$
E=E_{R}+\alpha E_{F},
$$

where $\alpha$ is a weighting parameter used to determine how much $E_{R}$ and $E_{F}$ individually contribute to the entire energy $E$. Its Gibbs form [11] is $P(Y=y \mid F=f)=$ $\frac{1}{Z} \exp \left[-\frac{1}{T} E\right]$, where $Z=\sum_{\Omega_{Y}} \exp \left[-\frac{1}{T} E\right]$ and $\Omega_{Y}$ is a set of all possible configurations of $Y$. Concrete forms for each of $E_{R}$ and $E_{F}$ are required for practical segmentation. Generally, for a segmentation task, the second order pairwise MLL model is chosen and the potentials of all non-pairwise cliques are defined to be zeros [11].

The intensity feature can be modelled using a Gamma distribution, allowing the energy form $E_{F}$ to be written as:

$$
E_{F}(x)=\sum_{s, Y_{s}=m}\left\{\frac{l}{\mu_{m}} x_{s}-(l-1) \log x_{s}+l \log \mu_{m}\right\}
$$

where $l$ is the number of looks. For the task of segmenting different ice types, the GLCP features are used as the image features. Under the assumption of a Gaussian distribution, the energy form $E_{F}$ of the product of all $p\left(f_{s}^{k} \mid Y_{s}=m\right)$ can be written as:

$$
E_{F}(f)=\sum_{s, Y_{s}=m}\left\{\sum_{k=1}^{K}\left[\frac{\left(f_{s}^{k}-\mu_{m}^{k}\right)^{2}}{2\left(\sigma_{m}^{k}\right)^{2}}+\log \left(\sqrt{2 \pi} \sigma_{m}^{k}\right)\right]\right\},
$$

where $\mu_{m}$ and $\sigma_{m}$ represent the class mean and standard deviation.

To implement the MRF model (Eq. (3)) requires estimation of three parameters: $\alpha$ (from Eq. (3)), $\mu_{m}$, and $\sigma_{m}$ (for all $\mathrm{m}$ ). Traditionally, estimation of $\mu_{m}$ and $\sigma_{m}$ for each class requires training data. However, using an unsupervised environment, training data are not provided. Instead, the expectation-maximization (EM) algorithm [12] [13] is used to estimate $\mu_{m}$ and $\sigma_{m}$.

The difficulty is that there is no closed-form definition for $\alpha$. A commonly used strategy [10] is to assign an a priori constant value by experience before executing the EM algorithm. A root problem is that the traditional MRF-based segmentation model is very easily trapped in local maxima due to the spatial homogeneity constraint imposed by the region labelling component. As a result, the feature modelling component might not be able to learn the global parameters (i.e. $\mu_{m}$ and $\sigma_{m}$ for each class). A new implementation scheme is proposed here to solve this problem by making the weighting parameter $\alpha$ vary during unsupervised segmentation. The introduction of the 
variable weighting parameter should not only enable the segmentation procedure to learn the global parameters of the feature modelling component but also impose a spatial homogeneity constraint on the label distribution (through the region labelling component). In this context, the parameter may vary with respect to the annealing procedure. The following function is selected for the variable weighting parameter $\alpha$ :

$$
\alpha(t)=c_{1} * \gamma^{t}+c_{2}, 0<\gamma<1,
$$

where $\gamma, c_{1}$ and $c_{2}$ are constants and $t$ represents the $t^{t h}$ iteration. Experimentally, we have determined that setting $\gamma=0.95, c_{1}=80$ and $c_{2}=1 / K$ (where $\mathrm{K}$ is the dimension of the feature space) are appropriate values for a variety of imagery. Using this function, the feature modelling component will first (when $\alpha(t)$ is larger) dominate the MRF model in order to learn its global parameters and then (when $\alpha(t)$ is close to $c_{2}$ ) interact with the region labelling component to refine the segmented result. Thus, the energy of the MRF model can be rewritten as:

$$
E=E_{R}+\alpha(t) E_{F}
$$

\section{EXPERIMENTAL RESULTS}

The image shown in Fig. 2(a) is part of a C-band $\mathrm{HH}$ RADARSAT ScanSAR image (100m pixel spacing) in the Baffin Bay / Davis Strait region acquired on February 7, 1998. This image consists of three types of sea ice: multiyear ice (bright floes), grey-white ice (running primarily from top to bottom in the middle), and grey ice (observed on the left hand side and surrounding the multi-year floes on the right hand side). Visually, this image would be very difficult to segment and even the manual segmentations by trained human operators would have tremendous variability (manual segmentation would also be a very time consuming exercise). Although tonal distinctions are noted visually, the histogram is unimodal (not shown).

Segmentation using K-means (Fig. 2(b)) shows a segmentation that is not effective. Boundaries of unique regions are somewhat defined, however, the regions themselves are "spotty" in their classification since local spatial interactions are not accounted. Segmentation using the MRF model with constant weighting produces an ineffective segmentation where the form of the ice regions is not properly recognized (Fig. 2(c)). The only method that produces an acceptable segmentation is the MRF model using a variable weighting scheme (Fig. 2(d)). Here, the multi-year floes are consistent and the grey and grey-white regions show acceptable divisions. The application of the MRF model with the variable parameter using a fused (texture and intensity) is able to generate the most accurate result.

\section{CONCLUSIONS}

An unsupervised means of segmenting SAR sea ice egg code regions is proposed and successfully tested. The variable weighting MRF procedure is a more appropriate method for performing SAR sea ice segmentation than using K-means or the traditional MRF method.

\section{ACKNOWLEDGMENTS}

The authors are thankful to GEOIDE (http://www.geoide.ulaval.ca/) and CRYSYS (http: //www.crysys.ca) for their financial support and CIS (http://www.cis.ec.gc.ca/) for providing the SAR sea ice images (all RADARSAT images (C)SA http://www.space.gc.ca).

\section{REFERENCES}

[1] D. A. Clausi, "An analysis of co-occurrence texture statistics as a function of grey level quantization," Canadian J. Remote Sensing, vol. 28, no. 1, pp. 1-18, 2002

[2] —, "Comparison and fusion of co-occurrence, Gabor and MRF texture features for classification of SAR sea-ice imagery," Atmosphere-Ocean, vol. 39, no. 3, pp. 183-194, 2000.

[3] L. K. Soh and C. Tsatsoulis, "Texture analysis of SAR sea ice imagery using gray level co-occurrence matrices," IEEE Trans. Geoscience and Remote Sensing, vol. 37, no. 2, pp. 780-795, 1999.

[4] D. G. Barber and E. F. LeDrew, "SAR sea ice discrimination using texture statistics: A multivariate approach," Photogrammetric Engineering and Remote Sensing, vol. 57, no. 4, pp. 385-395, 1991.

[5] M. Tuceryan and A. K. Jain, Handbook of Pattern Recognition and Computer Vision, Chapter 2: Texture Analysis. Singapore: World Scientific, 1993.

[6] R. M. Haralick, K. Shanmugam, and I. Dinstein, "Textural features for image classification," IEEE Trans. Sys. Man Cybern, vol. 3, pp. 610-621, 1973.

[7] A. Baraldi and F. Parmiggiani, "An investigation of the textural characteristic associated with gray level cooccurrence matrix statistical parameters," IEEE Trans. Geoscience and Remote Sensing, vol. 33, no. 2, pp. 293-304, 1995.

[8] R. O. Duda and P. E. Hart, Pattern Classification and Scene Analysis. A Wiley-Interscience Publication, USA, 2000.

[9] J. E. Besag, "Spatial interaction and the statistical analysis of lattice systems (with discussion)," J. Royal Stat. Soc., vol. B-36, pp. 192 236, 1974.

[10] S. Geman and D. Geman, "Stochastic relaxation, Gibbs distributions, and the Bayesian restoration of images," IEEE Trans. Pattern Anal. Machine Intell., vol. 6, no. 6, pp. 721-741, 1984.

[11] S. Z. Li, Markov Random Field Modeling in Computer Vision. New York: Springer-Verlag, 2001.

[12] A. P. Dempster, N. M. Laird, and D. B. Rubin, "Maximum likelihood from incomplete data via the EM algorithm," J. Royal Stat. Soc., vol. B-1, pp. 1-38, 1977.

[13] J. Zhang, "The mean field theory in EM procedures for Markov random fields," IEEE Trans. Signal Processing, vol. 40, no. 10, pp. 2570-2583, 1992. 


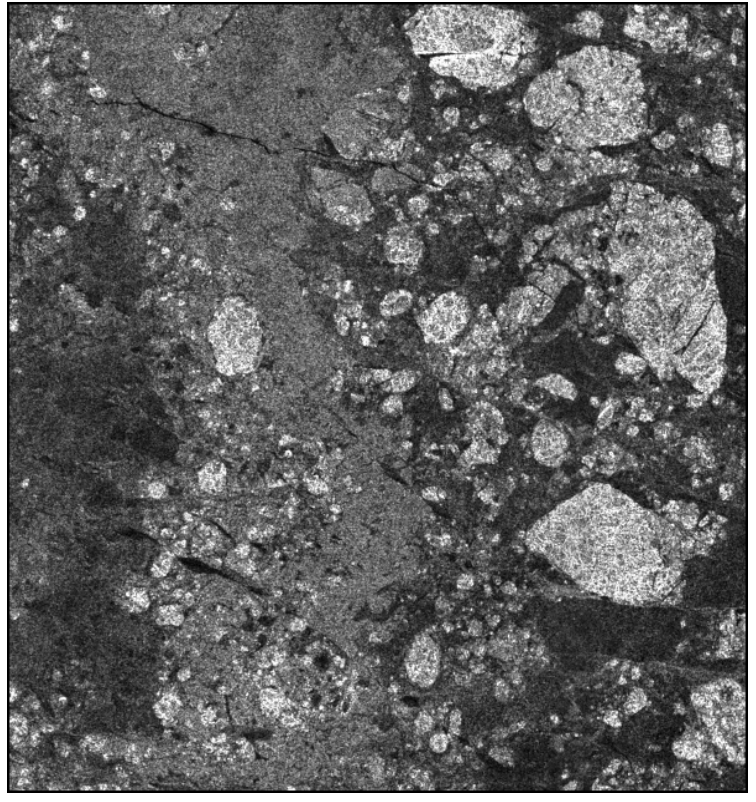

(a)

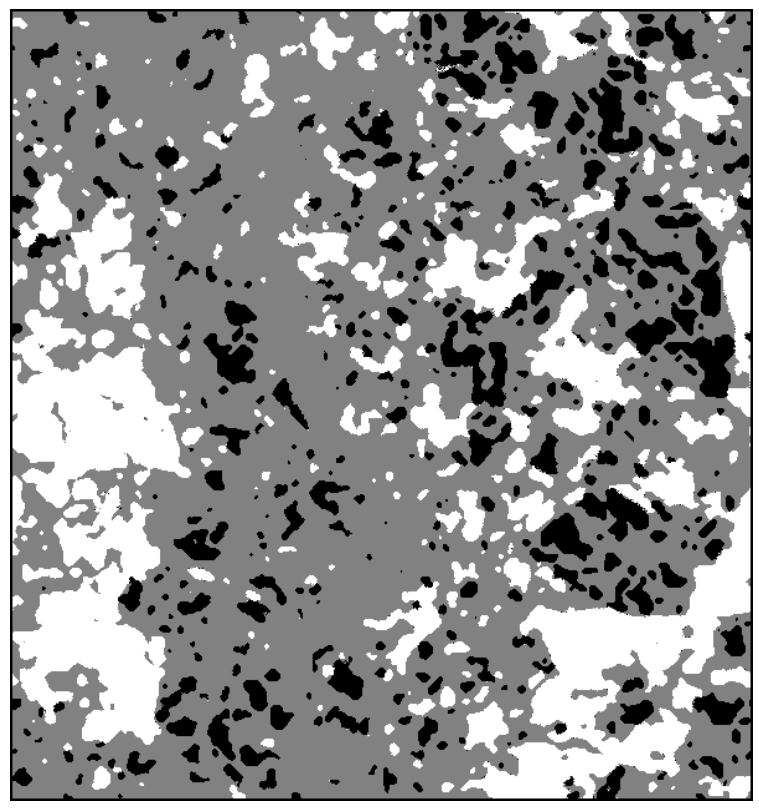

(c)

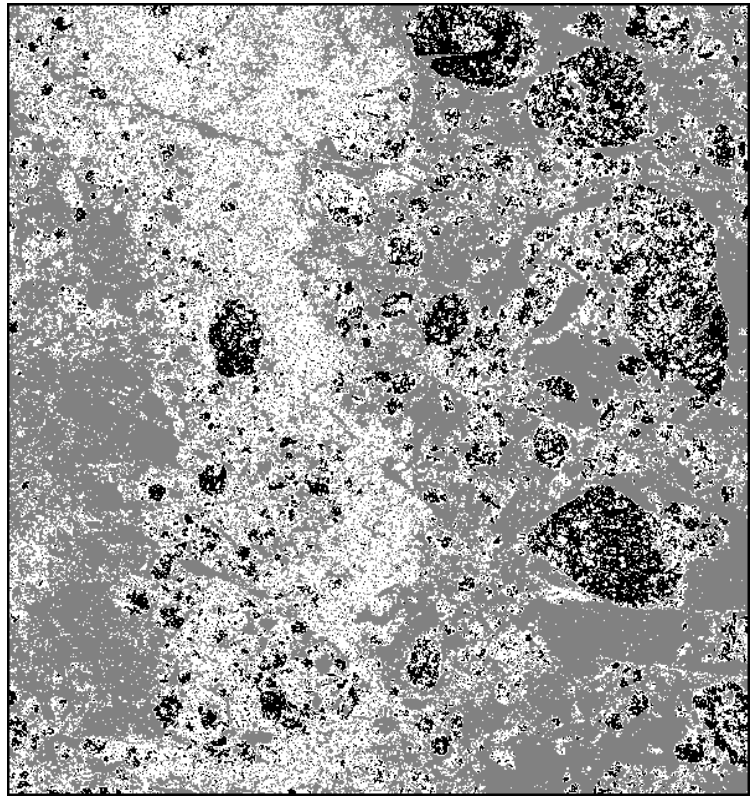

(b)

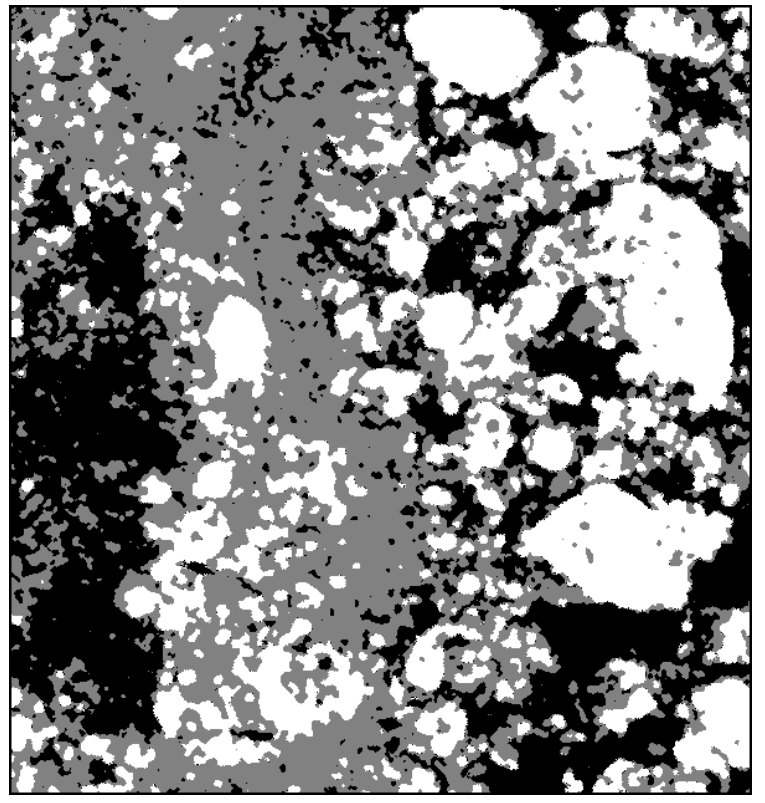

(d)

Fig. 2. (a) Original RADARSAT image $(631 \times 595$ pixels). Segmentation using (b) K-means, (c) MRF model with a constant weighting parameter $(\alpha=8)$ and (d) MRF model with a variable weighting parameter $\left(\alpha(t)=80 * 0.95^{t}+1 / 9\right)$. All segmentations performed using intensity fused with GLCP texture features. 